\section{Regulierte Selbstregulierung im frühen Interventions- und Sozialstaat}

Veranstalter: Peter Collin/Gerd Bender, Forschungsprojekt „Regulierte Selbstregulierung in rechtshistorischer Perspektive", MaxPlanck-Institut für europäische Rechtsgeschichte, Frankfurt am Main; Exzellenzcluster 243 „Die Herausbildung normativer Ordnungen“, Johann Wolfgang Goethe-Universität, Frankfurt am Main

Datum, Ort: 17.06.2010-19.06.2010, Bad Homburg

Bericht von: Maria Mercè Darnaculleta Gardella, Exzellenzcluster 16 „Kulturelle Grundlagen von Integration", Universität Konstanz

Vom 17. bis zum 19. Juni 2010 fand in Bad Homburg die zweite Sommertagung des Projekts „Regulierte Selbstregulierung in rechtshistorischer Perspektive“ des Max-PlanckInstituts für europäische Rechtsgeschichte statt. Mit dem Thema „Regulierte Selbstregulierung im frühen Interventions- und Sozialstaat" verbanden die Veranstalter das Ziel, historische Erscheinungsformen von staatlich gesteuerter und staatlich in Dienst genommener gesellschaftlicher Selbstorganisation $\mathrm{zu}$ eruieren und $\mathrm{zu}$ analysieren.

JÜRGEN SCHMIDT (Berlin) eröffnete das Themenspektrum mit einem Blick auf die Arbeiterbewegung des 19. Jahrhunderts. Die Arbeiterbewegung sei als eine vom Staat völlig unabhängige Bewegung entstanden und habe zahlreiche Aufgaben übernommen, die den Mechanismen der Selbstregulierung gehorchten. Schmidt führte dazu die Normen der Binnenorganisation der Arbeiterbewegung an, verwies aber auch auf die Verhaltenskodizes für das Sozialverhalten, die Festschreibung von Tugenden und Werten, die Subjektregulation in der Adaptation bürgerlicher Werte und die Streitkultur bei ungleich verteilten Gütern. Strittig bliebe, ob diese Selbstorganisationsformen sozialdemokratischer oder sozialistischer Tradition wegen ihrer wechselhaften Beziehungen zum Staat als regulierte Selbstregulierung beschrieben werden können. Denn schließlich sei der Staat zunächst gegen die Arbeiterbewegung vorgegangen und habe sich erst gegen Ende des 19. Jahrhunderts aktiv um die Regulierung der Arbeitsbeziehungen bemüht. Diese staatlichen Interventionen hätten zwar selbstregulierte Organisationen in den öffentlichen (kommunalen) Proporz integriert, dabei aber massiv in deren Strukturen eingegriffen.

CARSTEN KREMER (Frankfurt am Main) betonte die Rolle der Autonomie in der Selbstregulierung und präsentierte einen für die Rechtsquellenlehre höchst interessanten rechtshistorischen Aspekt der Bedeutung von Autonomie als Rechtsetzungsbefugnis. Gemeinden, Adel und andere Körperschaften, wie zum Beispiel Universitäten, seien Inhaber von so verstandener Autonomie gewesen, da sie in weiten Teilen die Freiheit der Selbstorganisation und Selbstnormierung unabhängig von staatlichen Eingriffen für sich in Anspruch nehmen konnten. Kremer konnte zeigen, wie die Anerkennung eines staatlichen Gesetzgebungsmonopols in der Staatsrechtslehre zu einer Debatte über die Autonomie als Rechtsquelle (originäre oder abgeleitete) geführt hatte und in welcher Weise die gegenwärtige Praxis zu der klassischen Diskussion in krassem Gegensatz stehe. Denn heute würden lediglich die Satzungen als Ergebnisse solch autonom-normativer Befugnisse anerkannt, nicht aber die Autonomie selbst als Rechtsquelle gesehen.

KATHRIN GROH (Speyer) analysierte, wie in der Weimarer Zeit der Verbandspluralismus zur Idee der Demokratie in Bezug gesetzt wurde. Sie betonte, alle Klassiker unter den demokratischen Staatsrechtslehrern von Hugo Preuß über Gerhard Anschütz, Richard Thoma bis zu Hermann Heller und Hans Kelsen - bezögen sich auf die Idee der Selbststeuerung. Auf die eine oder andere Weise kontrastierten sie aber auch alle die Vertretung und Aushandlung partikularer Interessen durch Berufsverbände mit der parlamentarischen Demokratie als Verkörperung der Allgemeinheit.

GERD BENDER (Frankfurt am Main) beschloss die erste Tagungssektion mit der Feststellung, die Regulierung von Arbeitsbedingungen sei der Bereich schlechthin, bei dem regulierte Selbstregulierung historisch in Erscheinung trete. In seinem Beitrag illustrierte er die Geschichte der Tarifautonomie an Hand konzeptioneller Fragestellungen der politischen Soziologie. Ausgehend von einer

Konstanzer Online-Publikations-System (KOPS)

URL: http://nbn-resolving.de/urn:nbn:de:bsz:352-145007

(C) H-Net, Clio-online, and the author, all rights reserved. 
systemtheoretischen Erfassung sozialer Beziehungen konnte er die Wechselwirkungen zwischen korporativen und staatlichen Interessen neu analysieren, wobei er die Tarifautonomie als ein ",mitverantwortetes, aber nicht verstaatlichtes Netzwerk" (in Form des NeoKorporatismus) beschrieb.

Das Referat von MATTHIAS SCHMOECKEL (Bonn) über die Rolle der Privatautonomie bei Bau und Betrieb der Eisenbahnen in Preußen zwischen 1830 und 1880 führte in die Sektion "Referenzgebiete" ein. Die Industrie habe in dieser Epoche ihre Freiheit vehement verteidigt und dadurch die Privatinitiative beim Eisenbahnbau gestärkt. Jedoch habe der wachsenden Notwendigkeit zur Erweiterung eines öffentlichen Transportnetzes die Ablösung des Patrimonialstaates durch einen Interventionsstaat korrespondiert. Schmoeckel sah Parallelen mit heutigen Tendenzen zur Etablierung von „Public Private Partnerships", da im Preußen des 19. Jahrhundert zwar das Schienennetz in öffentlicher Hand gelegen habe, die Betriebsleistungen aber aus Effizienzgründen von Privaten erbracht worden seien.

WOLFGANG AYAß (Kassel) analysierte das Gebiet der Unfallversicherungen unter dem Gesichtspunkt der Selbstregulierung. Mit dem Unfallversicherungsgesetz von 1880 habe eine Übertragung öffentlicher Aufgaben auf die Berufsgenossenschaften stattgefunden. In diesem Zusammenhang stellte er zwei Modi der Selbstregulierung heraus: erstens eine konfliktlösende Selbstregulierung, bei der die Berufsgenossenschaften als Schiedsgerichte tätig gewesen seien, und zweitens eine interne, organisatorische Selbstregulierung in den Berufsgenossenschaften. Diese und viele der folgenden Beiträge setzten Selbstverwaltung mit Selbstregulierung gleich, was mit einer bestimmten geschichtswissenschaftlichen Auffassung des Verhältnisses von Staat und Gesellschaft zusammenhängen dürfte.

PETER COLLIN (Frankfurt am Main) ging bei seiner Betrachtung kommunaler Selbstverwaltung als regulierter Selbstregulierung von einer traditionellen Auffassung aus, die kommunale Selbstverwaltung nicht der staatlichen, sondern der gesellschaftlichen Sphäre zurechnete. Aus dieser Perspektive thematisierte er die "Krise der kommunalen Selbst- verwaltung" in der Weimarer Republik. In dieser Zeit sei Selbstverwaltung vor allem auf Grund der Zuweisung von Fürsorgeaufgaben immer stärker technokratisch konturiert worden. Die rechtsdogmatischen und verwaltungswissenschaftlichen Debatten zu "Zentralisierung" und "Dezentralisierung" sowie zur Unterscheidung zwischen "eigenen" und „übertragenen“ Kommunalaufgaben würden somit auch eine Krise der Selbstregulierung reflektieren, wobei Selbstregulierung als Selbstbestimmungsmöglichkeit von Bürgern bzw. der sogenannten Zivilgesellschaft verstanden wurde.

ANDREAS THIER (Zürich) identifizierte in seinen Vortrag drei Dimensionen steuerrechtlich regulierender Einflüsse auf Prozesse gesellschaftlicher Selbstorganisation. Die erste Dimension beschreibe das Steuerrecht des 19. Jahrhunderts als Lenkungsinstrument von Prozessen und Sozialordnungen. In der zweiten Dimension werde am Beispiel des preußischen Dreiklassenwahlrechts das Steuerrecht zur Wahlrechtsverfassung in Beziehung gesetzt. Als drittes analysierte Thier die Steuergesetzgebung als Förderinstrument bestimmter Organisationsformen.

KARL CHRISTIAN FÜHRER (Hamburg) führte die Teilnehmer in die Riten und Risiken des Wohnungsmarktes des frühen 20. Jahrhunderts ein. Sein Ausgangspunkt war die traditionell schwache Stellung der Mieter gegenüber den Eigentümern vor dem ersten Weltkrieg. Nach Kriegsbeginn hatte man zur Beruhigung der sozialen Lage Mieteinigungsämter geschaffen. Diese hatten die Aufgabe, Konflikte zwischen Mietern und Vermietern zu lösen und ersetzten das gerichtliche Verfahren. Die Entscheidungen der Mieteinigungsämter seien tendenziell mieterfreundlich gewesen, vor allem aber für beide Parteien verbindlich. Allerdings existierten kaum rahmenrechtliche Vorgaben und Verfahrensrichtlinien für diese Schiedspraxis. Deshalb ließe sich das Verfahren auch als ein Beispiel „staatlich verordneter Selbstregulation ohne Garantien" bezeichnen.

DIETER SCHOTT (Darmstadt) berichtete über die „kooperative privatwirtschaftliche und kommunale Regulierung städtischer Infrastrukturen" von 1900 bis 1914 am Beispiel von Darmstadt und Mannheim. In bei- 
den Fällen betonte er die Rolle der Privatinitiativen und die variantenreichen Kooperationsformen zwischen Gemeinden und Privatunternehmern. Hier ließe sich bereits die Idee der Governance der Moderne erahnen - mit Elementen wie zum Beispiel kommunaler Einräumung von Straßenland, staatlichen Konzessionen und gemischtwirtschaftlichen Konsortien als Unternehmensform.

THOMAS BUCHER (Linz) beschrieb die Unbeständigkeit des Arbeitsmarktes im letzten Drittel des 19. Jahrhunderts und die Formen der Arbeitsvermittlung in der Zeit zwischen Kaiserreich und Weimarer Republik. In diesem Zeitraum sei eine Transformation der Akteure, der Regulierungsinstrumente und der Regulierungsobjekte zu beobachten, bei denen der Kommunalarbeitsnachweis eine große Rolle gespielt habe. Die Einbindung gesellschaftlicher Akteure, das heißt der Vertreter von Arbeitnehmer- und Arbeitgeberseite, in eine öffentlich-kommunale Schiedsgerichtsbarkeit und die Zentralität des Arbeitsmarkts als Regulierungsobjekt hätten dabei bedeutend zur Erfolgsgeschichte diese Nachweiswesens beigetragen.

VERA HIERHOLZER (Frankfurt am Main) thematisierte in ihrem Vortrag über die staatlich regulierte industrielle Selbstkontrolle in der Nahrungsmittelindustrie im 19. Jahrhundert einen auch in der gegenwärtigen Diskussion noch aktuellen Fall regulierter Selbstregulierung. Im Deutschen Kaiserreich seien Regulierung und Kontrolle von Nahrungsmitteln Bestandteil des Polizeirechtes gewesen, aber bereits als Verbraucherschutz und Nahrungsmittelschutz argumentativ implementiert worden. Mit dem Nahrungsmittelgesetz von 1879 habe sich die systematische Einbeziehung wissenschaftlicher Expertise und die Einrichtung von Gutachter- und Standardisierungsverfahren etabliert. Mit dem Deutschen Nahrungsmittelbuch, der Setzung von Qualitätsstands und der Implementierung von Qualitätsnachweisen sei eine Aufgabenverteilung etabliert worden, bei der der Staat die Minimalstandards festgelegt habe, deren inhaltliche Ausgestaltung und Kontrolle der Wissenschaft oblag. Die Wirtschaft als dritte Partei habe korrigierend in den Prozess eingreifen können und die Qualitätsstandards ihrerseits zur werbenden Zertifizierung der
Waren genutzt.

WILFRIED RUDLOFF (Kassel) widmete den letzten Vortrag den Beratungsgremien im Staatsapparat des Kaiserreichs und der Weimarer Republik. Diese periodisch zusammentretenden Gremien seien mehrheitlich mit nichtstaatlichen Akteuren besetzt gewesen und qua Verwaltungsakt in die Lage versetzt worden, nach selbst- oder vorgegebenen Verfahrensregeln politik- bzw. verwaltungsberatend tätig zu werden. Die Gremien, zum Beispiel der Kolonialrat, hätten sich oft von gesellschaftlichen Partikularinteressen leiten lassen, sie waren oft mit Verbandsvertretern besetzt. Inwieweit diese Beratungstätigkeit die staatliche Entscheidungsfindung beeinflusste, müsste differenziert beurteilt werden. Die zentrale Frage laute dabei, ob bzw. in welchem Ausmaß es sich bei diesem Modus staatlich-privatverbandlicher Interaktion um eine Okkupation der Normsetzungsautorität des Staates durch private und societale Akteure jenseits verfassungsmäßig vorgesehener Beteiligungsorgane handelte.

In der Einleitung zur Abschlussdiskussion gelang es Peter Collin, die heterogenen Vortagsthemen wieder zusammenzuführen und sie als historische Muster dessen zu einen, was heute regulierte Selbstregulierung genannt wird. Der Tagung gelang ein Überblick über die verschiedenen Regulierungs- und Selbstregulierungsmodi, es konnte das Kontinuum zwischen Normdurchsetzung und Selbstkontrollmechanismen abgebildet und in Beziehungen $\mathrm{zu}$ unterschiedlichen Ordnungsregimes gesetzt werden. Dabei standen mal kooperative, mal rein private Selbstregulierungen im Fokus, mal rückte der Gegensatz zwischen formellen und informellen Selbstregulierungsmustern ins Zentrum der Analysen. Unterstrichen wurde vor allem noch einmal, dass staatliche Instrumentalisierung gesellschaftlicher Selbstregulierung als indirekter Mechanismus zur Erreichung öffentlicher Ziele eine Konstante in der Genese des Sozialstaates ist. Gleichermaßen konnten die Vorläufer der aktuellen Legitimationsprobleme sozialer Selbstregulierung historisch fundiert werden und ursächliche Gründe in der Rechtsquellentheorie ausgemacht werden. Dennoch kommen bei der praktizierten Verwendung des Begriffes Selbstre- 
gulierung Zweifel an der Nützlichkeit dieser Kategorie auf, da sie sowohl regulierte Selbstregulierung als auch reine Selbstregulierung unter sich $\mathrm{zu}$ vereinen scheint. Man konnte beobachten, dass sich eine ungenaue Gleichsetzung von Selbstregulierung sowohl mit rein interner Selbstorganisation (Körperschaften, Gewerkschaften) als auch mit öffentlich-privater Kooperation (Beispiel Eisenbahn), der Selbstbestimmung in kommunalen Verwaltungsstrukturen und auch der Autonomie breit machte. Dabei kamen viele Beispiele zur Sprache, die mit dem zeitgenössischen rechtlichen Modus der regulierten Selbstregulierung sehr viel mehr zu tun haben, so etwa die normsetzende Selbstregulierung in der Industrie und bei Tarifverträgen, die zertifizierende und wissenschaftliche Standards abbildende Selbstregulierung in der Nahrungsmittelbranche sowie die konfliktlösende Selbstregulierung bei Schiedsgerichten, Berufsgenossenschaften oder Mieteinigungsämtern. Die große Aufmerksamkeit, die der Rolle des Staates gewidmet wurde, lassen Anschlüsse an die gegenwärtigen Forschungen der Rechtswissenschaft und Politikwissenschaft erhoffen, wenn es um die notwendige Revision des Staatsverständnisses geht. Insofern bot die Tagung wichtige Einblicke in das gar nicht so Selbstverständliche. Was noch aussteht, ist eine historisch fundierte Differenzierung zwischen der spontanen regulierten Selbstregulierung, die im Nachhinein in staatliche Steuerungskonzepte integriert wird, und der bewussten Initiierung regulierter Selbstregulierung von Seiten des Staates. Ferner empfiehlt es sich in Zukunft, die historische Analyse des Verhältnisses von öffentlichem und Privatrecht stärker unter dem Aspekt der regulierten Selbstregulierung vorzunehmen, dies kann auch wegweisend sein für eine vergleichende Betrachtung auf europäischer Ebene. Allein, die Selbstregulierungsforschung steckt in Rechtswissenschaft und Rechtsgeschichte noch in den Kinderschuhen, aber das hohe Niveau und die hervorragende Komposition der Tagung haben deutlich gemacht, dass es kein Nischen-, sondern ein Zukunftsthema der Rechtsgeschichte sein wird.

\section{Konferenzübersicht:}

1. Sektion: Schlüsseldiskurse in Recht und Politik

Jürgen Schmidt (Berlin): Regulierte Selbstregulierung und Arbeiterbewegung im 19. Jahrhundert

Carsten Kremer (Frankfurt am Main) :Autonomie als Rechtsquelle. Die Diskussion über nicht-staatliche Rechtssetzungsbefugnisse in der Rechtswissenschaft des 19. Jahrhunderts

Kathrin Groh (Speyer): Regulierte Selbstregulierung in der demokratischen Staatslehre der Weimarer Zeit

Gerd Bender (Frankfurt am Main): Regulierte Selbstregulierung und das Korporatismusproblem

Matthias Schmoeckel (Bonn): Die Ideengeschichte der Regulierung im 19. und beginnenden 20. Jahrhundert. Das Beispiel der Eisenbahn

\section{Sektion: Referenzgebiete des Rechts}

Wolfgang Ayaß (Kassel): Selbstregulierung in der gesetzlichen Unfallversicherung

Peter Collin (Frankfurt am Main): Kommunalrecht unter staatlichem Regulierungsdruck in der Weimarer Zeit

Andreas Thier (Zürich): Steuerung gesellschaftlicher Selbstorganisation durch Steuerrecht

\section{Sektion: Praxisfelder}

Karl Christian Führer (Hamburg): Privatstaatliche Regulierung knapper Ressourcen die Wohnungswirtschaft

Dieter Schott (Darmstadt): Kooperative privatwirtschaftliche und kommunale Regulierung städtischer Infrastrukturen. Die Beispiele Darmstadt und Mannheim 1900-1914

Thomas Buchner (Linz): Wer reguliert welchen Markt? Arbeitsvermittlung zwischen Kaiserreich und Weimarer Republik

Vera Hierholzer (Frankfurt am Main): Staatlich regulierte industrielle Selbstnormierung und Selbstkontrolle

Wilfried Rudloff (Kassel): Politikberatung Politikbeeinflussung - Selbstnormierung 
Tagungsbericht Regulierte Selbstregulierung im frühen Interventions- und Sozialstaat. 17.06.2010-19.06.2010, Bad Homburg, in: H-Soz-u-Kult 09.09.2010. 